\title{
PERFORMANCE OF PROTOTYPES AND START UP OF SERIES FABRICATION OF THE LHC ARC QUADRUPOLES
}

\author{
J.Billan, V.Remondino, A.Siemko, N.Smirnov, T.Tortschanoff, \\ CERN, Geneva, Switzerland \\ M.Peyrot, J.M.Rifflet, F.Simon, CEA Saclay, France
}

\begin{abstract}
The construction of three prototype arc quadrupoles for the LHC machine has been concluded successfully. These magnets underwent warm and cold magnetic measurements as well as many other tests, both in CEASaclay's laboratory and at CERN. Their training qualifies them for use in the LHC machine and their measured field quality points to only very minor corrections. An excellent correlation is found between warm and cold magnetic measurements. The prototype quadrupole design has been fully retained for the series fabrication of the 400 magnets and their cold masses by industry. This paper describes the main tests and measurement results of all three prototypes. It further explains the logistics for the manufacturing of the series of cold masses. These cold masses contain not only the main quadrupole but also different combinations of corrector magnets. Thus, together with variants imposed by the cryogenic configuration of the machine, 40 different types of cold masses have to be fabricated by the firm, to which the contract has been adjudicated.
\end{abstract}

\section{INTRODUCTION}

The main quadrupole magnets for the arcs of the LHC have been described in earlier publications [1], [2]. Some preliminary test results have also been presented elsewhere [3]. The objective of this paper is to give a summary picture of the construction and tests of all three prototypes and to explain the provisions for the series fabrication.

The design and the construction of these twin-aperture magnets are the result of a close collaboration between CERN and the CEA-Saclay laboratory in France. This collaboration concerns the design, prototyping and technology transfer to industry as well as the following up of the series fabrication by CEA. The mounting of the quadrupoles into their cryostats was executed in a collaboration between CERN and the CNRS laboratory of Orsay in France [4].

The twin-aperture quadrupole magnets are housed in a cold mass together with the arc corrector magnets. On one end the dipole-sextupole correctors are mounted, on the other end, the connection end, either the octupole, respectively the tuning or skew quadrupole correctors. The vessel of the cold mass functions as the stiffening element of the assembly and at the same time as helium pressure vessel.

Table 1 recalls the main parameters of these magnets, for more details see [3]. The three prototype units, named SSS3, SSS4 and SSS5, have been constructed in Saclay. (SSS stands for Short Straight Section.) While SSS3 and SSS4 were assembled together with all their corrector magnets, the corrector magnets inside the cold mass of SSS5 were simulated by dummy masses. All quadrupoles were submitted to warm magnetic measurements at CEASaclay. After delivery and assembly into their horizontal cryostats, the first two of them, SSS3 and SSS4, have undergone cold tests and magnetic measurements at CERN. The third one, SSS5, has been tested at the cryogenic test station in CEA-Saclay before being delivered to CERN. SSS3 and SSS4 have been integrated into the second LHC test line, called "String 2", where all electrical, cryogenic and vacuum functions will be tested [5].

Table 1: Main parameters and characteristics of the LHC quadrupole magnet and cold mass

\begin{tabular}{|l|c|c|}
\hline & Value & Unit \\
\hline $\begin{array}{l}\text { Injection field gradient }(0.45 \\
\text { TeV beam energy) }\end{array}$ & 14.5 & $\mathrm{~T} / \mathrm{m}$ \\
\hline $\begin{array}{l}\text { Nominal field gradient }(7 \mathrm{TeV} \\
\text { beam energy) }\end{array}$ & 223 & $\mathrm{~T} / \mathrm{m}$ \\
\hline Nominal current & $11^{\prime} 870$ & $\mathrm{~A}$ \\
\hline Operating temperature & 1.9 & $\mathrm{~K}$ \\
\hline Magnetic length at 1.9 K & 3.1 & $\mathrm{~m}$ \\
\hline $\begin{array}{l}\text { Cold mass length at 293 K, } \\
\text { between end covers }\end{array}$ & 5355 & $\mathrm{~mm}$ \\
\hline Cold mass weight & 6.5 & $\mathrm{~T}$ \\
\hline
\end{tabular}

\section{WARM MAGNETIC MEASUREMENTS}

Warm magnetic measurements were performed in each aperture after collaring and providing the inter-coil connections. They were repeated in both apertures once the cold masses were finished. They have been described in [3] together with their results in terms of integrated multipoles components. Fig. 1 shows one collared aperture on CEA's station for the warm magnetic measurements. 


\section{COLD TESTS AND TRAINING}

All three cold masses were pressure tested up to 2.6 $\mathrm{MPa}$, and leak tested in a vessel at CEA. Fig. 2 shows the insertion of a cold mass into its pressure test vessel.

Table 2: Multipole components measured at nominal excitation for SSS3, SSS4 and SSS5.

\begin{tabular}{|c|c|c|c|c|c|c|}
\hline \multirow{2}{*}{\begin{tabular}{|l} 
Meas. at: \\
Multipole
\end{tabular}} & CERN & $\begin{array}{l}11870 \mathrm{~A} \\
\mathbf{R N}\end{array}$ & $\begin{array}{r}\text { SSS4 } \\
\text { CI }\end{array}$ & CERN & SSS5 & $\begin{array}{l}11870 \mathrm{~A} \\
\mathbf{E A}\end{array}$ \\
\hline & Left & Right & Left & Right & Left & Right \\
\hline b3 & 4.406 & -1.117 & -0.650 & 0.345 & 0.934 & 0.589 \\
\hline b4 & -0.043 & -0.017 & -0.324 & -0.004 & -0.100 & 0.049 \\
\hline b5 & -0.753 & -1.023 & 0.155 & -0.198 & -0.218 & 0.058 \\
\hline b6 & 6.674 & 7.374 & 6.600 & 6.314 & 6.330 & 6.178 \\
\hline b7 & .039 & -0.093 & -0.090 & -0.032 & 0.209 & 0.253 \\
\hline b8 & -0.034 & 0.007 & 0.010 & 0.009 & -0.160 & -0.033 \\
\hline b9 & -0.007 & 0.017 & -0.017 & -0.010 & 0.006 & -0.016 \\
\hline b10 & -0.124 & -0.112 & -0.165 & -0.221 & -0.260 & -0.202 \\
\hline $\mathbf{a} 3$ & 3.899 & -2.604 & -1.616 & -1.616 & 1.533 & -1.109 \\
\hline a4 & -2.920 & -1.363 & 2.725 & 2.725 & -0.748 & -4.179 \\
\hline a5 & 0.319 & 0.751 & 0.002 & 0.002 & 0.511 & 0.180 \\
\hline a6 & 0.313 & 0.276 & 0.412 & 0.219 & 0.325 & 0.212 \\
\hline a7 & 0.041 & -0.256 & 0.104 & -0.087 & 0.044 & -0.018 \\
\hline a8 & -0.023 & 0.017 & -0.070 & 0.132 & 0.000 & -0.161 \\
\hline a9 & 0.033 & -0.014 & 0.006 & 0.006 & 0.005 & -0.011 \\
\hline $\mathbf{a 1 0}$ & 0.051 & 0.029 & 0.079 & 0.051 & 0.067 & 0.068 \\
\hline
\end{tabular}

SSS3 and SSS4 were tested at CERN's cryogenic test facility for the LHC magnets. They underwent a thorough verification and test programme, including thermal cycles and detailed magnetic measurements.

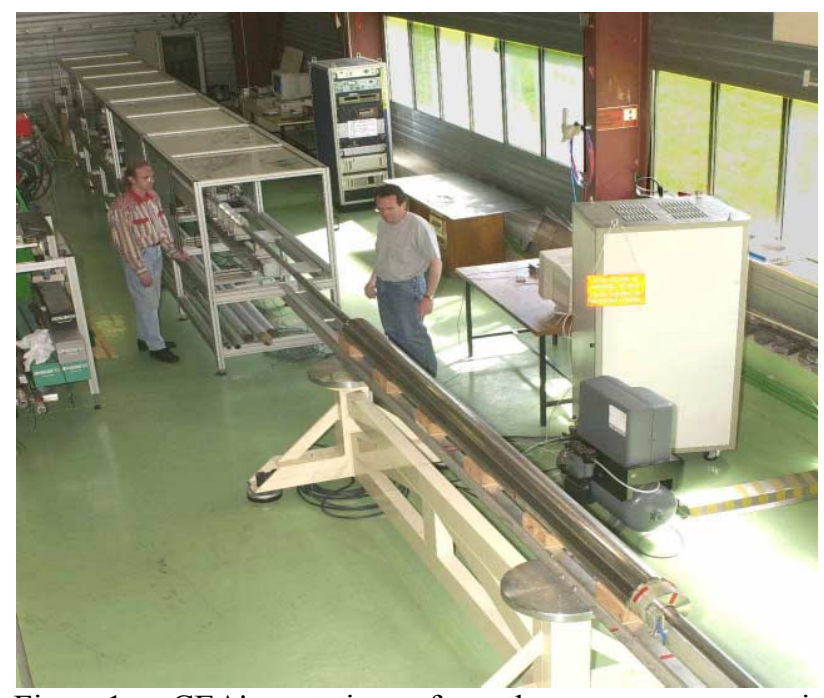

Fig. 1: CEA's station for the warm magnetic measurements with a collared aperture.

Fig. 3 shows the quench history of all three quadrupole magnets. Two of them, SSS3 and SSS5, had one quench below the nominal excitation, SSS4 behaved better since its very first quench appeared well above the nominal value.
Similar to what was measured at room temperature conditions, the measured multipole components at the nominal excitation, show a re-assuring consistency between the apertures with the exception of the apertures of the first magnet, SSS3, see Table 2. This may be explained by the fact that due to initial technical assembly problems the coils had to be collared several times.

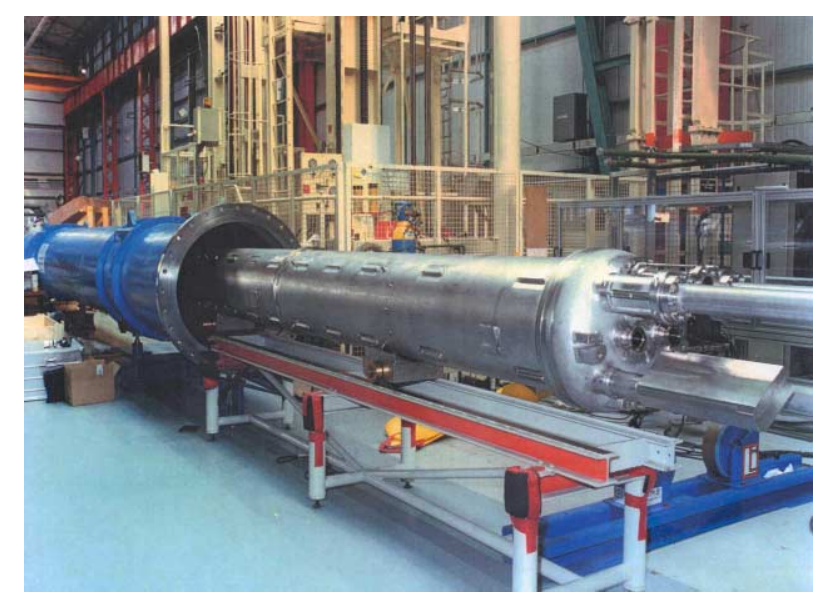

Fig. 2: Insertion of SSS cold mass into the pressure test vessel at CEA-Saclay.

The influence of the persistent currents at the excitation of beam injection was measured and compared to the computed ones [6]. Table 3 summarises this for the dodeca-pole and the twenty-pole components, $b_{6}$ and $b_{10}$, in the straight part of the apertures in all three prototypes.

Table 3: Influence of persistent currents on $b_{6}$ and $b_{10}$ at injection, $760 \mathrm{~A}$, in straight part, in units of 1.E-4 at $17 \mathrm{~mm}$

\begin{tabular}{|l|c|c|c|c|}
\hline & \multicolumn{2}{|c|}{$\mathrm{b}_{6}$} & \multicolumn{2}{c|}{$\mathrm{b}_{10}$} \\
Measured & Ap. 1 & Ap. 2 & Ap. 1 & Ap. 2 \\
\hline SSS3 & -3.30 & -3.30 & 0.1 & -0.1 \\
\hline SSS4 & -3.29 & -3.57 & 0.1 & 0.1 \\
\hline SSS5 & -3.71 & -3.70 & 0.1 & 0.1 \\
\hline Computed & \multicolumn{2}{|c|}{-3.48} & \multicolumn{2}{|c|}{0.1} \\
\hline
\end{tabular}

The variation among the six apertures can be explained by the use of the super-conducting cable originating from the pre-series manufacturing of different suppliers.

\section{START-UP OF SERIES FABRICATION}

CERN issued a detailed technical specification for the construction of the quadrupole cold masses. A number of components are separately ordered and delivered by CERN and include the following items:

- super-conducting cables,

- polyimide insulation, both for the conductor and ground insulation,

- austenitic steel for the collars,

- low-carbon steel for the yoke laminations, 
- bus-bars,

- quench protection diodes,

- quench heaters,

- cold bore tubes,

- heat exchanger tubes,

- corrector magnets,

- instrumentation and its wires,

- interconnection bellows.

Most of these items are ordered for both the LHC dipoles and quadrupoles. In this way CERN achieves considerable savings because of quantity prices and by avoiding third party profits.

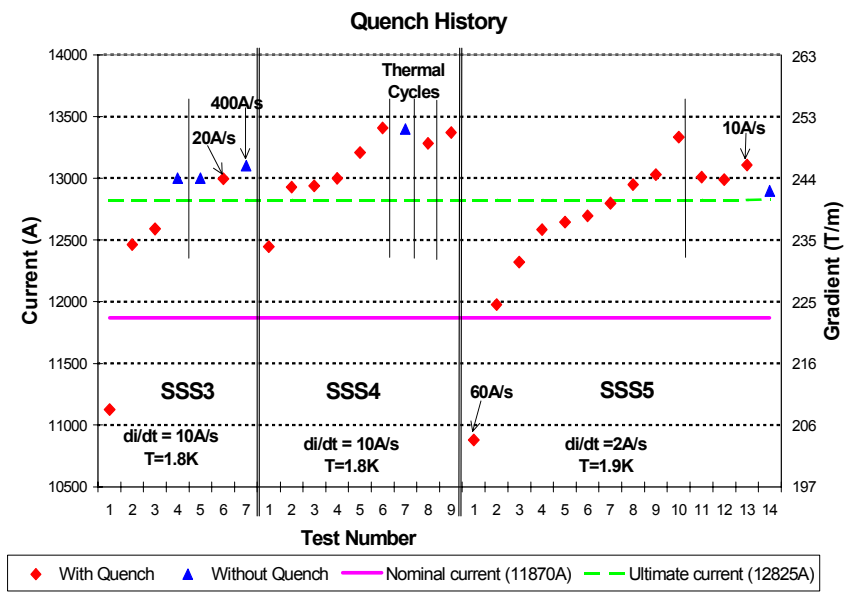

Fig. 3: Quench history of SSS3, SSS4, and SSS5.

The call for tender was answered by seven firms in five European states. The contract was adjudicated to the German company ACCEL Instruments and the contract was signed in July 2000. The order concerns 360 fully assembled cold masses, which come in 40 different versions, depending on the quadrupole polarities, the busbar and diode versions and the various cryogenic equipment. To this eight additional cold masses were added to be delivered in the form of spare parts. This will allow, in the case of need, to assemble the correct combination of corrector magnets with the other components of the cold mass. On top of this, the order includes 32 quadrupole magnets to be integrated into special cold masses still to be designed for the LHC dispersion suppressor regions.

The manufacturer has prepared two factory halls of in total $4200 \mathrm{~m}^{2}$ for the series fabrication of these units. One of these halls is equipped with a 5 m-deep pit, which will house the tooling allowing to assemble the cold masses vertically. Some of the tooling used in CEA-Saclay for the prototype fabrication is being taken over for usage in this factory. CEA's technicians, experienced from their involvement in the prototype work, will ensure the technology transfer and the following-up during the series fabrication.

The multiple sub-supplies for the cold mass fabrication require a sophisticated co-ordination and thorough quality control. This is presently in preparation for both, the ones provided by CERN and those which the main contractor has to procure by himself. The preparation work also concerns the manufacturer's own tooling which he has to provide in order to achieve the scheduled fabrication rate. This rate is supposed to reach four cold masses per week, after a certain learning and start-up period. The cryostating of the cold masses in industry, by another firm, will have to follow at the same rate. This will condition all operations required for the cold tests and measurements at $\mathrm{CERN}$ and thereafter the final preparations for installing these units into the LHC tunnel at CERN. Since only a limited buffer storage capacity is available the sequence of fabrication must closely follow the sequence of installation into the tunnel of the machine.

\section{REFERENCES}

[1] T. Tortschanoff et al., "The Short Straight Sections for the LHC", Proc. PAC97 Conference, Vancouver, Canada. 1997.

[2] M. Peyrot, J.M. Rifflet, F. Simon, T. Tortschanoff, P. Vedrine, "Construction of the New Prototype of Main Quadrupole Cold Masses for the Arc Short Straight Sections of LHC", Proc. MT-16, Ponte Vedra Beach, Florida, USA, 1999.

[3] T. Tortschanoff, J. Billan, L. Bottura, V. Remondino, A. Siemko, M. Peyrot, J.M. Rifflet, F. Simon, "Performance of Series-design Prototype Main Quadrupoles for the LHC", Proc. EPAC-2000, Vienna, 2000

[4] L. Nielsen, V. Parma, T. Renaglia, P. Rohmig, T. Tortschanoff, J.-B. Bergot, Ph. Dambre, E. Roy, D. Vincent, M. Peyrot, J.-M. Rifflet, F. Simon, "A Modular Design for the 56 Variants of the Short Straight Section in the Arcs of the Large Hadron Collider (LHC)", Proc. EPAC-2000, Vienna, 2000.

[5] R. Saban et al., "Assembly and Precommissioning of the LHC Test String 2", Proc. PAC2001, Chicago, 2001.

[6] Rob Wolf, S. Le Nour, "The Expected Persistent Current Field Errors in the LHC Main Dipole and Quadrupole", LHC Project Note 230, private communication, Aug. 2000. 